\title{
Genetic similarity between Pagurus gracilipes (Stimpson, 1858) (Decapoda: Paguroidea) and another pagurid and diogenid hermit crabs from Russian waters of the Sea of Japan
}

\author{
N.I. Zaslavskaya, E.S. Kornienko, O.M. Korn \\ A.V. Zhirmunsky Institute of Marine Biology, Far East Branch, Russian Academy of Sciences, 17 \\ Palchevskogo Str., Vladivostok 690041, Russia. \\ e-mail:nad_zas@mail.ru
}

ABSTRACT: Biochemical genetic approach was applied to clarify the taxonomic status of the hermit crab Pagurus gracilipes which was compared with five species of the family Paguridae ( $P$. brachiomastus, $P$. ochotensis, $P$. middendorffii, $P$. proximus, $P$. minutus) and with two species of the family Diogenidae (Diogenes nitidimanus and Stratiotes nigroapiculus) using 18 allozyme loci. Genetic identity values between $P$. gracilipes and species of the family Paguridae (from $0.611 \pm 0.117$ for $P$. gracilipes vs $P$. proximus, to $0.249 \pm 0.102$ for $P$. gracilipes vs $P$. middendorffii) suggest that this species should retain its position in the genus Pagurus. This conclusion is supported by similar average identity value between other species of the genus Pagurus $(I=0.444 \pm 0.110)$ and low genetic similarity between the genera Diogenes and Stratiotes $(I=0.167 \pm 0.084)$ and between the families Paguridae and Diogenidae $(I=0.102 \pm 0.005)$.

KEY WORDS: Decapoda; hermit crab; allozyme electrophoresis; genetic similarity; Sea of Japan.

\section{Генетическое сходство между Pagurus gracilipes (Stimpson, 1858) (Decapoda: Paguroidea) и другими видами раков-отшельников из российских вод Японского моря}

\author{
Н.И. Заславская, Е.С. Корниенко, О.М. Корн \\ Институт биологии моря им. А.В. Жирмунского ДВО РАН, ул. Пальчевского, 17, Владивос- \\ ток 690041 Россия. \\ e-mail:nad_zas@mail.ru \\ РЕЗЮМЕ: Для уточнения таксономического статуса Pagurus gracilipes использова- \\ ны методы биохимической генетики. Этот вид сравнили с пятью видами семейства \\ Paguridae (P. brachiomastus, $P$. ochotensis, $P$. middendorffi, $P$. proximus, $P$. minutus) и \\ двумя видами семейства Diogenidae (Diogenes nitidimanus и Stratiotes nigroapiculus)
}

Printed in 2010 
по 18 аллозимным локусам. Значения генетического сходства, полученные при сравнении $P$. gracilipes с другими видами семейства Paguridae (от $0.611 \pm 0.117$ с $P$. proximus до $0.249 \pm 0.102$ с P. middendorffii), свидетельствуют о принадлежности этого вида к роду Pagurus. Этот вывод подтверждается близкими значениями генетического сходства между другими видами рода Pagurus (в среднем $I=0.444 \pm$ $0.110)$ и низкими значениями генетического сходства между родами Diogenes и Stratiotes $(I=0.167 \pm 0.084)$, а также семействами Paguridae и Diogenidae $(I=0.102 \pm 0.005)$.

КЛЮЧЕВЫЕ СЛОВА: Decapoda; раки-отшельники, аллозимный электрофорез, генетическое сходство, Японское море.

\section{Introduction}

The hermit crab Pagurus gracilipes (Stimpson, 1858) is known from the northern Japan, including Hokkaido and northeastern Honshu, and from the continental coast of the Russian Far East, where it inhabits subtidal zones to 42 m depth (Komai, 1998).

The taxonomic position of this species is subject to scientific discussion. A new genus Parapagurodes has been established by McLaughlin and Haig (1973). In 1998, four Japanese species of Pagurus, including $P$. gracilipes, have been transferred to Parapagurodes on the basis of morphology of sexual tubes in males (Komai, 1998). However, later P. gracilipes has been returned to Pagurus (McLaughlin, Asakura, 2004).

Allozyme electrophoresis was successfully used in resolving taxonomy of decapod crustacean species (Hedgecock et al., 1982; Fuseya, Watanabe, 1996; Stewart, Cook, 1998; Mia et al., 1999; Gouws, Stewart, 2000; Austin, Ryan, 2002).

The aim of this study was to apply biochemical genetics to clarify the taxonomic position of $P$. gracilipes among the Paguridae. To evaluate the degree of genetic difference/similarity between species within a genus, genera within a family and between families, five pagurid and two diogenid species inhabiting Peter the Great Bay (Sea of Japan) and having the stable taxonomic position were also analyzed using enzyme electrophoresis.

\section{Material and methods}

\section{Sample collection}

Samples of Pagurus gracilipes (Stimpson, 1858 ) as well as $P$. ochotensis Brandt, $1851, P$. brachiomastus (Thallwitz, 1892), P. proximus Komai, 2000, P. minutus Hess, 1865, P. middendorffii Brandt, 1851 (Paguridae), Diogenes nitidimanus Terao, 1913, and Stratiotes nigroapiculus Komai, 2009 (Diogenidae) were collected at depths from 0.5 to $5 \mathrm{~m}$ in Vostok Bay (Peter the Great Bay, Sea of Japan) in June 2008. Sample sizes are given in Table 1.

\section{Enzyme electrophoresis}

Two tissues (liver and muscle) from each animal were homogenized together in one vial, and were further used for electrophoretic analysis. Horizontal electrophoresis was carried out using 13\% starch gels as described in Zaslavskaya (1989). Three continuous buffer systems were used to resolve 13 enzymes: (1) TEB (trisEDTA-boric acid, $\mathrm{pH}$ 8.5), (2) TC (tris-citric acid, $\mathrm{pH}$ 7.0) and (3) TM (tris-maleic acid, $\mathrm{pH}$ 7.4). Electrophoretic buffer systems and enzyme systems used in the analysis are listed below (enzyme abbreviations, enzyme classification numbers, and isozyme locus standard abbreviations are given in parentheses). TEB was used to resolve vial glucose phosphate isomerase (GPI; EC 4.3.1.5, Gpi), glutamate pyruvate transaminase (GPT; EC 2.6.1.2, Gpt), glutation reductase (GR; EC 1.6.4.2, Gr), hexo- 
Table 1. Allele frequencies at 18 allozyme loci in eight hermit crabs ( $\mathrm{N}$ is the sample size). Таблица 1. Частоты аллелей в 18 аллозимных локусах у 8 видов раков-отшельников $(\mathrm{N}$ - размер выборки).

\begin{tabular}{|c|c|c|c|c|c|c|c|c|c|}
\hline \multirow[b]{2}{*}{ Locus } & \multirow[b]{2}{*}{ Allele } & \multicolumn{8}{|c|}{ Species } \\
\hline & & 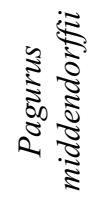 & 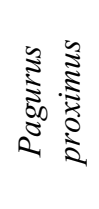 & 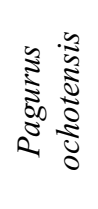 & 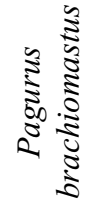 & 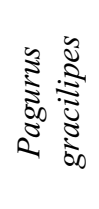 & 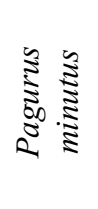 & 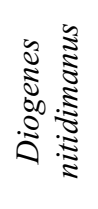 & 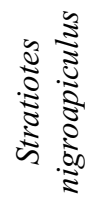 \\
\hline 1 & 2 & 3 & 4 & 5 & 6 & 7 & 8 & 9 & 10 \\
\hline \multicolumn{10}{|l|}{ Gpi } \\
\hline & $(\mathrm{N})$ & 17 & 17 & 18 & 15 & 29 & 18 & 20 & 9 \\
\hline & 1 & 0.000 & 0.000 & 0.000 & 0.000 & 0.000 & 0.056 & 0.000 & 0.000 \\
\hline & 2 & 0.118 & 0.088 & 0.000 & 0.000 & 0.000 & 0.944 & 0.000 & 0.000 \\
\hline & 3 & 0.000 & 0.059 & 0.306 & 0.000 & 0.000 & 0.000 & 0.000 & 0.000 \\
\hline & 4 & 0.323 & 0.853 & 0.000 & 0.067 & 0.897 & 0.000 & 0.000 & 0.000 \\
\hline & 5 & 0.059 & 0.000 & 0.694 & 0.000 & 0.000 & 0.000 & 0.000 & 0.000 \\
\hline & 6 & 0.000 & 0.000 & 0.000 & 0.000 & 0.000 & 0.000 & 0.000 & 0.000 \\
\hline & 7 & 0.500 & 0.000 & 0.000 & 0.900 & 0.103 & 0.000 & 0.000 & 0.000 \\
\hline & 8 & 0.000 & 0.000 & 0.000 & 0.033 & 0.000 & 0.000 & 0.000 & 1.000 \\
\hline & 9 & 0.000 & 0.000 & 0.000 & 0.000 & 0.000 & 0.000 & 1.000 & 0.000 \\
\hline \multicolumn{10}{|l|}{$M p i$} \\
\hline & $(\mathrm{N})$ & 18 & 17 & 19 & 18 & 29 & 18 & 20 & 8 \\
\hline & 1 & 0.000 & 0.000 & 0.000 & 0.000 & 0.000 & 0.000 & 0.025 & 0.000 \\
\hline & 2 & 0.000 & 0.000 & 0.000 & 0.000 & 1.000 & 0.000 & 0.000 & 0.000 \\
\hline & 3 & 0.000 & 0.000 & 0.000 & 0.000 & 0.000 & 0.000 & 0.050 & 0.000 \\
\hline & 4 & 0.000 & 0.000 & 1.000 & 0.000 & 0.000 & 0.944 & 0.000 & 0.000 \\
\hline & 5 & 0.972 & 0.941 & 0.000 & 0.611 & 0.000 & 0.056 & 0.150 & 0.000 \\
\hline & 6 & 0.028 & 0.059 & 0.000 & 0.389 & 0.000 & 0.000 & 0.275 & 0.000 \\
\hline & 7 & 0.000 & 0.000 & 0.000 & 0.000 & 0.000 & 0.000 & 0.200 & 0.000 \\
\hline & 8 & 0.000 & 0.000 & 0.000 & 0.000 & 0.000 & 0.000 & 0.150 & 0.000 \\
\hline & 9 & 0.000 & 0.000 & 0.000 & 0.000 & 0.000 & 0.000 & 0.150 & 1.000 \\
\hline \multicolumn{10}{|l|}{$G p t$} \\
\hline & $(\mathrm{N})$ & 18 & 17 & 19 & 18 & 29 & 18 & 20 & \\
\hline & 1 & 0.000 & 0.059 & 0.000 & 0.139 & 0.000 & 0.000 & 0.000 & 0.000 \\
\hline & 2 & 0.000 & 0.941 & 0.000 & 0.778 & 0.983 & 1.000 & 0.000 & 0.000 \\
\hline & 3 & 0.194 & 0.000 & 0.000 & 0.083 & 0.017 & 0.000 & 0.025 & 0.125 \\
\hline & 4 & 0.112 & 0.000 & 0.579 & 0.000 & 0.000 & 0.000 & 0.000 & 0.000 \\
\hline & 5 & 0.000 & 0.000 & 0.026 & 0.000 & 0.000 & 0.000 & 0.775 & 0.000 \\
\hline & 6 & 0.694 & 0.000 & 0.395 & 0.000 & 0.000 & 0.000 & 0.125 & 0.750 \\
\hline & 7 & 0.000 & 0.000 & 0.000 & 0.000 & 0.000 & 0.000 & 0.075 & 0.125 \\
\hline \multicolumn{10}{|l|}{$I p p$} \\
\hline & (N) & 16 & 17 & 19 & 18 & 26 & 16 & 20 & 8 \\
\hline & 1 & 0.031 & 0.000 & 0.000 & 0.000 & 0.000 & 0.000 & 1.000 & 0.000 \\
\hline & 2 & 0.031 & 0.618 & 1.000 & 1.000 & 1.000 & 1.000 & 0.000 & 1.000 \\
\hline & 3 & 0.750 & 0.147 & 0.000 & 0.000 & 0.000 & 0.000 & 0.000 & 0.000 \\
\hline & 4 & 0.157 & 0.206 & 0.000 & 0.000 & 0.000 & 0.000 & 0.000 & 0.000 \\
\hline & 5 & 0.031 & 0.029 & 0.000 & 0.000 & 0.000 & 0.000 & 0.000 & 0.000 \\
\hline
\end{tabular}


Table 1 (contituing)

Таблица 1 (продолжение)

\begin{tabular}{|c|c|c|c|c|c|c|c|c|c|}
\hline 1 & 2 & 3 & 4 & 5 & 6 & 7 & 8 & 9 & 10 \\
\hline \multicolumn{10}{|l|}{$M d h-1$} \\
\hline & $(\mathrm{N})$ & 18 & 17 & 19 & 18 & 26 & 18 & 20 & 8 \\
\hline & 1 & 0.000 & 0.000 & 0.000 & 0.000 & 1.000 & 0.000 & 0.000 & 0.000 \\
\hline & 2 & 1.000 & 1.000 & 1.000 & 1.000 & 0.000 & 1.000 & 0.175 & 0.000 \\
\hline & 3 & 0.000 & 0.000 & 0.000 & 0.000 & 0.000 & 0.000 & 0.775 & 0.000 \\
\hline & 4 & 0.000 & 0.000 & 0.000 & 0.000 & 0.000 & 0.000 & 0.000 & 1.000 \\
\hline & 5 & 0.000 & 0.000 & 0.000 & 0.000 & 0.000 & 0.000 & 0.050 & 0.000 \\
\hline \multicolumn{10}{|l|}{$M d h-2$} \\
\hline & $(\mathrm{N})$ & 10 & 9 & 11 & 10 & 19 & 10 & 8 & 8 \\
\hline & 1 & 0.000 & 0.000 & 0.000 & 0.000 & 0.000 & 0.000 & 0.000 & 0.000 \\
\hline & 2 & 0.000 & 0.000 & 0.000 & 1.000 & 1.000 & 0.000 & 0.000 & 0.000 \\
\hline & 3 & 1.000 & 0.000 & 1.000 & 0.000 & 0.000 & 0.000 & 0.000 & 0.000 \\
\hline & 4 & 0.000 & 1.000 & 0.000 & 0.000 & 0.000 & 1.000 & 0.000 & 0.000 \\
\hline & 5 & 0.000 & 0.000 & 0.000 & 0.000 & 0.000 & 0.000 & 0.000 & 1.000 \\
\hline & 6 & 0.000 & 0.000 & 0.000 & 0.000 & 0.000 & 0.000 & 1.000 & 0.000 \\
\hline \multicolumn{10}{|l|}{ Got-1 } \\
\hline & $(\mathrm{N})$ & 18 & 17 & 19 & 18 & 29 & 18 & 20 & 8 \\
\hline & 1 & 0.000 & 0.000 & 0.000 & 0.167 & 0.000 & 0.000 & 0.000 & 0.000 \\
\hline & 2 & 0.000 & 0.000 & 0.000 & 0.000 & 0.000 & 0.000 & 0.950 & 0.000 \\
\hline & 3 & 0.000 & 0.000 & 0.000 & 0.000 & 0.000 & 0.000 & 0.000 & 0.938 \\
\hline & 4 & 0.194 & 0.000 & 0.026 & 0.000 & 0.000 & 0.000 & 0.000 & 0.000 \\
\hline & 5 & 0.000 & 0.000 & 0.000 & 0.000 & 0.000 & 1.000 & 0.000 & 0.000 \\
\hline & 6 & 0.806 & 0.971 & 0.974 & 0.000 & 1.000 & 0.000 & 0.050 & 0.000 \\
\hline & 7 & 0.000 & 0.029 & 0.000 & 0.000 & 0.000 & 0.000 & 0.000 & 0.062 \\
\hline & 8 & 0.000 & 0.000 & 0.000 & 0.833 & 0.000 & 0.000 & 0.000 & 0.000 \\
\hline \multicolumn{10}{|l|}{ Got-2 } \\
\hline & $(\mathrm{N})$ & 18 & 17 & 19 & 18 & 29 & 18 & 20 & 8 \\
\hline & 1 & 1.000 & 0.971 & 1.000 & 0.000 & 0.000 & 0.000 & 0.000 & 1.000 \\
\hline & 2 & 0.000 & 0.029 & 0.000 & 1.000 & 1.000 & 1.000 & 0.000 & 0.000 \\
\hline & 3 & 0.000 & 0.000 & 0.000 & 0.000 & 0.000 & 0.000 & 0.000 & 0.000 \\
\hline & 4 & 0.000 & 0.000 & 0.000 & 0.000 & 0.000 & 0.000 & 1.000 & 0.000 \\
\hline \multicolumn{10}{|l|}{$G r$} \\
\hline & $(\mathrm{N})$ & 18 & 17 & 19 & 18 & 29 & 18 & 20 & 8 \\
\hline & 1 & 0.000 & 0.000 & 0.000 & 0.000 & 0.000 & 1.000 & 1.000 & 0.938 \\
\hline & 2 & 1.000 & 0.000 & 0.000 & 0.000 & 0.000 & 0.000 & 0.000 & 0.062 \\
\hline & 3 & 0.000 & 0.000 & 0.000 & 0.972 & 0.000 & 0.000 & 0.000 & 0.000 \\
\hline & 4 & 0.000 & 1.000 & 1.000 & 0.028 & 1.000 & 0.000 & 0.000 & 0.000 \\
\hline \multicolumn{10}{|l|}{$P g m$} \\
\hline & $(\mathrm{N})$ & 18 & 17 & 19 & 18 & 29 & 18 & 20 & 8 \\
\hline & 1 & 0.000 & 0.000 & 0.000 & 0.000 & 0.000 & 0.000 & 0.000 & 1.000 \\
\hline & 2 & 0.000 & 0.000 & 0.000 & 0.027 & 0.586 & 0.000 & 0.000 & 0.000 \\
\hline & 3 & 0.000 & 0.000 & 0.027 & 0.000 & 0.000 & 0.056 & 0.000 & 0.000 \\
\hline & 4 & 0.000 & 0.000 & 0.000 & 0.000 & 0.000 & 0.944 & 0.100 & 0.000 \\
\hline & 5 & 0.000 & 0.000 & 0.000 & 0.056 & 0.414 & 0.000 & 0.000 & 0.000 \\
\hline & 6 & 1.000 & 0.971 & 0.947 & 0.0917 & 0.000 & 0.000 & 0.000 & 0.000 \\
\hline & 7 & 0.000 & 0.029 & 0.026 & 0000 & 0000 & 0000 & 0.900 & 0.000 \\
\hline
\end{tabular}


Table 1 (contituing)

Таблица 1 (продолжение)

\begin{tabular}{|c|c|c|c|c|c|c|c|c|c|}
\hline 1 & 2 & 3 & 4 & 5 & 6 & 7 & 8 & 9 & 10 \\
\hline \multicolumn{10}{|l|}{ Aldh } \\
\hline & $(\mathrm{N})$ & 12 & 12 & 14 & 13 & 24 & 12 & 15 & 8 \\
\hline & 1 & 0.000 & 0.000 & 0.000 & 1.000 & 0.000 & 0.000 & 1.000 & 0.000 \\
\hline & 2 & 0.000 & 0.000 & 0.000 & 0.000 & 0.000 & 0.000 & 0.000 & 1.000 \\
\hline & 3 & 0.000 & 0.000 & 0.071 & 0.000 & 0.062 & 0.000 & 0.000 & 0.000 \\
\hline & 4 & 0.917 & 1.000 & 0.929 & 0.000 & 0.938 & 0.000 & 0.000 & 0.000 \\
\hline & 5 & 0.000 & 0.000 & 0.000 & 0.000 & 0.000 & 0.000 & 0.000 & 0.000 \\
\hline & 6 & 0.083 & 0.000 & 0.000 & 0.000 & 0.000 & 0.042 & 0.000 & 0.000 \\
\hline & 7 & 0.000 & 0.000 & 0.000 & 0.000 & 0.000 & 0.958 & 0.000 & 0.000 \\
\hline \multicolumn{10}{|l|}{$H k$} \\
\hline & $(\mathrm{N})$ & 8 & 8 & 8 & 8 & 10 & 8 & 10 & 8 \\
\hline & 1 & 0.000 & 0.000 & 0.000 & 0.000 & 0.050 & 0.875 & 0.850 & 1.000 \\
\hline & 2 & 1.000 & 1.000 & 1.000 & 1.000 & 0.950 & 0.125 & 0.000 & 0.000 \\
\hline & 3 & 0.000 & 0.000 & 0.000 & 0.000 & 0.000 & 0.000 & 0.000 & 0.000 \\
\hline & 4 & 0.000 & 0.000 & 0.000 & 0.000 & 0.000 & 0.000 & 0.150 & 0.000 \\
\hline \multicolumn{10}{|l|}{$A c-1$} \\
\hline & $(\mathrm{N})$ & 8 & 8 & 8 & 8 & 10 & 8 & 10 & 8 \\
\hline & 1 & 0.000 & 0.000 & 0.000 & 0.000 & 0.000 & 0.000 & 0.000 & 1.000 \\
\hline & 2 & 0.000 & 1.000 & 1.000 & 1.000 & 1.000 & 1.000 & 0.000 & 0.000 \\
\hline & 3 & 0.000 & 0.000 & 0.000 & 0.000 & 0.000 & 0.000 & 0.300 & 0.000 \\
\hline & 4 & 0.000 & 0.000 & 0.000 & 0.000 & 0.000 & 0.000 & 0.700 & 0.000 \\
\hline & 5 & 0.000 & 0.000 & 0.000 & 0.000 & 0.000 & 0.000 & 0.000 & 0.000 \\
\hline & 6 & 1.000 & 0.000 & 0.000 & 0.000 & 0.000 & 0.000 & 0.000 & 0.000 \\
\hline \multicolumn{10}{|l|}{$A c-2$} \\
\hline & $(\mathrm{N})$ & 8 & 8 & 8 & 8 & 10 & 8 & 10 & 8 \\
\hline & 1 & 0.000 & 0.000 & 0.000 & 0.000 & 0.000 & 0.000 & 1.000 & 0.000 \\
\hline & 2 & 0.000 & 0.062 & 0.000 & 0.000 & 0.000 & 0.000 & 0.000 & 0.000 \\
\hline & 3 & 0.000 & 0.938 & 1.000 & 1.000 & 1.000 & 1.000 & 0.000 & 0.000 \\
\hline & 4 & 0.000 & 0.000 & 0.000 & 0.000 & 0.000 & 0.000 & 0.000 & 1.000 \\
\hline & 5 & 0.000 & 0.000 & 0.000 & 0.000 & 0.000 & 0.000 & 0.000 & 0.000 \\
\hline & 6 & 1.000 & 0.000 & 0.000 & 0.000 & 0.000 & 0.000 & 0.000 & 0.000 \\
\hline \multicolumn{10}{|l|}{ Lap } \\
\hline & $(\mathrm{N})$ & 14 & 17 & 18 & 17 & 28 & 18 & 10 & 8 \\
\hline & 1 & 0.000 & 0.000 & 0.056 & 0.000 & 0.018 & 0.000 & 1.000 & 0.000 \\
\hline & 2 & 0.000 & 0.000 & 0.111 & 0.000 & 0.803 & 0.000 & 0.000 & 0.000 \\
\hline & 3 & 0.000 & 0.000 & 0.694 & 0.000 & 0.000 & 0.000 & 0.000 & 0.000 \\
\hline & 4 & 0.000 & 0.000 & 0.000 & 0.000 & 0.179 & 0.000 & 0.000 & 0.000 \\
\hline & 5 & 0.000 & 0.676 & 0.139 & 0.118 & 0.000 & 0.611 & 0.000 & 0.000 \\
\hline & 6 & 0.036 & 0.324 & 0.000 & 0.000 & 0.000 & 0.361 & 0.000 & 0.000 \\
\hline & 7 & 0.357 & 0.000 & 0.000 & 0.000 & 0.000 & 0.028 & 0.000 & 0.812 \\
\hline & 8 & 0.607 & 0.000 & 0.000 & 0.676 & 0.000 & 0.000 & 0.000 & 0.188 \\
\hline & 9 & 0.000 & 0.000 & 0.000 & 0.206 & 0.000 & 0.000 & 0.000 & 0.000 \\
\hline \multicolumn{10}{|l|}{ Est-1 } \\
\hline & $(\mathrm{N})$ & 8 & 8 & 8 & 9 & 14 & 8 & 10 & 8 \\
\hline & 1 & 1.000 & 1.000 & 0.000 & 0.000 & 0.000 & 0.000 & 0.000 & 0.000 \\
\hline & 2 & 0.000 & 0.000 & 1.000 & 0.833 & 0.000 & 0.063 & 0.000 & 0.000 \\
\hline
\end{tabular}


Table 1 (contituing) Таблица 1 (продолжение)

\begin{tabular}{|c|c|c|c|c|c|c|c|c|c|}
\hline $\mathbf{1}$ & $\mathbf{2}$ & $\mathbf{3}$ & $\mathbf{4}$ & $\mathbf{5}$ & $\mathbf{6}$ & $\mathbf{7}$ & $\mathbf{8}$ & $\mathbf{9}$ & $\mathbf{1 0}$ \\
\hline & 3 & 0.000 & 0.000 & 0.000 & 0.167 & 1.000 & 0.063 & 0.000 & 0.000 \\
\hline & 4 & 0.000 & 0.000 & 0.000 & 0.000 & 0.000 & 0.874 & 0.000 & 0.000 \\
\hline & 5 & 0.000 & 0.000 & 0.000 & 0.000 & 0.000 & 0.000 & 0.000 & 0.063 \\
\hline & 6 & 0.000 & 0.000 & 0.000 & 0.000 & 0.000 & 0.000 & 0.600 & 0.874 \\
\hline & 7 & 0.000 & 0.000 & 0.000 & 0.000 & 0.000 & 0.000 & 0.400 & 0.063 \\
\hline Est-2 & & & & & & & & & \\
\hline & $(\mathrm{N})$ & 8 & 8 & 8 & 9 & 14 & 8 & 10 & 8 \\
\hline & 1 & 0.000 & 0.000 & 0.000 & 0.000 & 0.000 & 0.000 & 0.000 & 0.000 \\
\hline & 2 & 1.000 & 0.000 & 0.000 & 0.000 & 0.000 & 0.000 & 0.000 & 0.000 \\
\hline & 3 & 0.000 & 0.812 & 1.000 & 1.000 & 1.000 & 1.000 & 0.000 & 0.000 \\
\hline & 4 & 0.000 & 0.188 & 0.000 & 0.000 & 0.000 & 0.000 & 0.000 & 0.000 \\
\hline & 5 & 0.000 & 0.000 & 0.000 & 0.000 & 0.000 & 0.000 & 0.600 & 0.000 \\
\hline & 6 & 0.000 & 0.000 & 0.000 & 0.000 & 0.000 & 0.000 & 0.400 & 0.000 \\
\hline & 7 & 0.000 & 0.000 & 0.000 & 0.000 & 0.000 & 0.000 & 0.000 & 1.000 \\
\hline Est-3 & & & & & & & & & \\
\hline & $(\mathrm{N})$ & 10 & 9 & 11 & 10 & 19 & 10 & 10 & 8 \\
\hline & 1 & 0.000 & 0.000 & 0.000 & 0.000 & 0.000 & 0.000 & 1.000 & 0.000 \\
\hline & 2 & 0.000 & 0.000 & 0.000 & 1.000 & 0.000 & 0.000 & 0.000 & 0.000 \\
\hline & 3 & 1.000 & 1.000 & 1.000 & 0.000 & 1.000 & 0.000 & 0.000 & 1.000 \\
\hline & 4 & 0.000 & 0.000 & 0.000 & 0.000 & 0.000 & 1.000 & 0.000 & 0.000 \\
\hline
\end{tabular}

kinase (Hk; EC 2.7.1.1, Hk), inorganic pyrophosphatase (IPP; EC 3.6.1.1, Ipp), nonspecific esterases (EST; EC 3.1.1..., Est-1, Est-2, Est3). TC was used to resolve acid phosphatase (Ap;EC 3.1.3.2, Ac-1, Ac-2), leucine aminopeptidase (LAP; EC 3.4.11.1, Lap), malate dehydrogenase (MDH; EC 1.1.1.37, Mdh-1, Mdh2), glutamate oxaloacetate transaminase (GOT; EC 2.6.1.1, Got-1, Got-2). TM was used to resolve alanopine dehydrogenase (ALPDH; EC 1.5.1.17, Aldh), mannose phosphate isomerase (MPI; EC 5.3.1.8, Mpi), phosphoglucomutase (PGM; EC 2.7.5.1, Pgm). Genetic interpretation of electrophoretic data was based on specific patterns of enzyme bands on zymograms (Harris, Hopkinson, 1976; Manchenko, 1994).

\section{Data analysis}

Allele frequencies were calculated using the program BIOSYS (Swofford, Selander, 1981). Nei's (1978) unbiased genetic identity $(I)$ and genetic distance $(D)$ coefficients, estimation of their standard error were calculated using the program DBOOT (Pudovkin et al., 1996). Phe- nogram was constructed by the unweighted pair group method from estimates of Nei's (1978) genetic similarity using software package NTSYS (Rohlf, 1988).

\section{Results and discussion}

Allele frequencies at 18 allozyme loci coding for 13 enzyme systems calculated for all species are given in Table 1. When it was possible to test fits to Hardy-Weinberg equilibria, no significant deviations from expectations were found. Nei's (1978) genetic similarity and distance are shown in Table 2.

Figure 1 shows the dendrogram of genetic relationships between all studied species. All six species of the genus Pagurus (including $P$. gracilipes) form a cluster with an average $I$ value of $0.444 \pm 0.110$. Two genera, Diogenes and Stratiotes, and two families, Paguridae and Diogenidae, exhibited much lower values of genetic identity $(I=0.167 \pm 0.084$ and $I=0.102 \pm$ 0.005 , respectively). Among the pagurids, $P$. gracilipes is rather closely related to $P$. proxi- 
Table 2. Nei's (1978) genetic similarity (above diagonal) and distance (below diagonal) among eight hermit crabs inhabiting Peter the Great Bay.

Таблица 2. Значения генетического сходства (выше диагонали) и расстояния (ниже диагонали)

(Nei, 1978) для 8 видов раков-отшельников, обитающих в заливе Петра Великого.

\begin{tabular}{|l|c|c|c|c|c|c|c|c|}
\hline \multicolumn{1}{|c|}{ Species } & 1 & 2 & 3 & 4 & 5 & 6 & 7 & 8 \\
\hline 1. Pagurus middendorffii & $* * * *$ & 0.577 & 0.506 & 0.287 & 0.249 & 0.084 & 0.033 & 0.190 \\
\hline 2. Pagurus proximus & 0.549 & $* * * *$ & 0.700 & 0.495 & 0.611 & 0.439 & 0.027 & 0.159 \\
\hline 3. Pagurus ochotensis & 0.682 & 0.356 & $* * * *$ & 0.486 & 0.539 & 0.379 & 0.024 & 0.200 \\
\hline 4.Pagurus brachiomastus & 1.249 & 0.704 & 0.722 & $* * * *$ & 0.501 & 0.439 & 0.092 & 0.072 \\
\hline 5. Pagurus gracilipes & 1.392 & 0.493 & 0.618 & 0.692 & $* * * *$ & 0.372 & 0.007 & 0.123 \\
\hline 6. Pagurus minutus & 2.473 & 0.823 & 0.972 & 0.824 & 0.988 & $* * * *$ & 0.131 & 0.170 \\
\hline 7. Diogenes nitidimanus & 3.410 & 3.613 & 3.715 & 2.390 & 4.942 & 2.035 & $* * * *$ & 0.167 \\
\hline 8. Stratiotes nigroapiculus & 1.663 & 1.839 & 1.611 & 2.627 & 2.099 & 1.772 & 1.790 & $* * * *$ \\
\hline
\end{tabular}

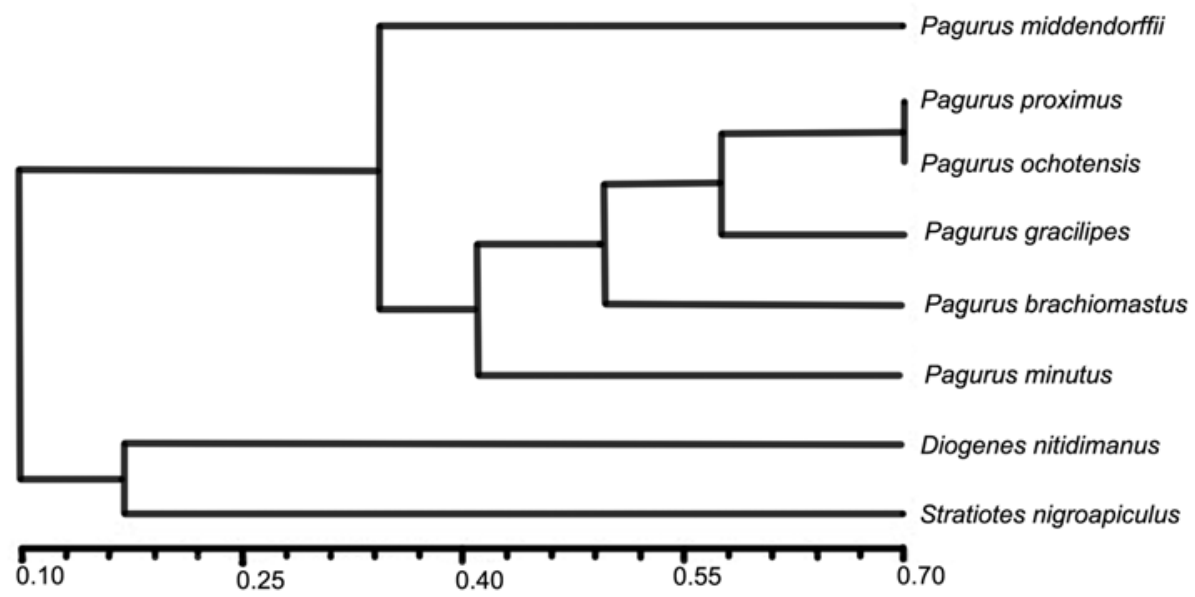

Fig. 1. UPGMA tree constructed on the base of genetic similarity (Nei, 1978) showing genetic relationships among eight hermit crab species.

Рис. 1. UPGMA-дерево, построенное на основе индексов генетического сходства (Nei, 1978), демонстрирующее генетические связи между 8 видами раков-отшельников.

mus $(I=0.611 \pm 0.117), P$. ochotensis $(I=0.539 \pm$ $0.121)$ and $P$. brachiomastus $(I=0.501 \pm 0.117)$.

It was shown that the average genetic similarity between the pagurid species inhabiting Peter the Great Bay is lower than the average genetical identity obtained by Hedgecock et al. (1982) for 26 pairs of congeneric decapod species $(I=0.66 \pm 0.15)$ and values obtained by Mulley and Latter (1980) for the genera Metapenaeus $(I=0.69 \pm 0.08)$ and Penaeus $(I=0.65 \pm$ 0.08 ) and within the genus Scylla (Gao, Watanabe, 1998). Earlier, we have found that two spider crabs (Pisoides bidentatus and Pugettia quadridens) and two intertidal crabs (Hemigrapsus sanguineus and $H$. penicillatus) were also very similar genetically (Zaslavskaya et al., 2007). Genetic identity values between these pairs of species were high $(I=0.758 \pm 0.092$ and $I=0.821 \pm 0.078$, respectively). Fairly low genetic identity among the pagurid species is in line with the observed variability in morphology among adults and among larvae within the genus Pagurus. This genus contains more than a 100 species (Ingle, 1985). Because of their recognized heterogeneity, species of the genus Pagurus were repeatedly divided into informal 
groups based on either adult or larval morphology. For example, four groups (A-D) were distinguished based on the similarities of larval characters (see Roberts, 1970; McLaughlin, Gore, 1988).

According to larval characters, most of the pagurids inhabiting Russian waters of the Sea of Japan (P. brachiomastus, $P$. ochotensis, $P$. middendorffii, $P$. proximus and $P$. minutus) are members of Group A (typical representative is Pagurus bernhardus) (Kornienko, Korn, 2006). Larval characters of $P$. gracilipes considerably differ from larval characters of these pagurids (Kornienko, Korn, 2007). Detailed description indicated that this species should rather be placed in Group C (typical representative is Pagurus anachoretus). Therefore, larval morphology confirms that $P$. gracilipes differs from the other pagurids living in Peter the Great Bay.

However, according to genetic relationships, $P$. gracilipes undoubtedly belongs to the genus Pagurus. This conclusion is supported by similar identity values between other species of the genus Pagurus and much lower genetic similarities between the genera Diogenes and Stratiotes and between the families Paguridae and Diogenidae. Genetic identity values among the pagurid species in our study are close to interspecific values obtained in electrophoretic studies of other invertebrates. These studies suggest that $85 \%$ of $I$-values between congeneric species exceed 0.35 , while between genera fall below 0.35 (Thorpe, 1982; Thorpe, Solé-Cava, 1994).

\section{Acknowledgements}

The project was supported by the Russian Foundation for Fundamental Research (grant no. 08-04-929). We are greatly indebted to Dr Tomoyuki Komai (Natural History Museum and Institute, Chiba, Japan) for the identification of Diogenes nitidimanus and Stratiotes nigroapiculus and Dr Akira Asakura (Natural History Museum and Institute, Chiba, Japan) for offering helpful comments.

\section{References}

Austin C.M., Ryan S.R. 2002. Allozyme evidence for a new species of freshwater crayfish of the genus Cherax Erichson (Decapoda: Parastacidae) from the southwest of Western Australia // Invertebrate Systematics. Vol.16. P.357-367.

Fuseya R., Watanabe S. 1996. Genetic variability in the mud crab genus Scylla (Brachyura: Portunidae) // Fisheries Science. Vol.62. P.705-709.

Gouws G., Stewart B.A. 2001. Potamonautid river crabs (Decapoda, Brachyura, Potamonautidae) of KwaZulu-Natal, South Africa // Water SA. Vol.27. No.1. P.85-98.

Harris H., Hopkinson D.A. 1976. Handbook of enzyme electrophoresis in human genetics. North-Holland, Amsterdam.

Hedgecock D., Tracey M., Nelson K. 1982. Genetics // D.E. Bliss (ed.). The biology of Crustacea. Vol.2. Embryology, morphology, and genetics. New York: Academic Press. P.283-403.

Ingle R.W. 1985. Northeastern Atlantic and Mediterranien hermit crabs (Crustacea: Anomura: Paguroidea: Paguridae). I. The genus Pagurus Fabricius, 1775 // Journal of Natural History. Vol.19. P.745-769.

Komai T. 1998. The taxonomic position of Pagurus gracilipes (Stimpson, (1858) and Pagurus nipponensis (Yokoya, 1933), and description of a new species of Pagurus (Decapoda, Anomura, Paguridae) from Japan // Zoosystema. Vol.20. P.265-288.

Kornienko E.S., Korn O.M. 2006. The larval development of Pagurus proximus (Decapoda: Anomura: Paguridae) reared in the laboratory // Journal of the Marine Biological Association of the United Kingdom. Vol.86. P.369-381.

Kornienko E.S., Korn O.M. 2007. Larval development of the hermit crab Pagurus gracilipes (Stimpson, 1858) (Decapoda: Anomura: Paguridae) reared in the laboratory // Invertebrate Reproduction and Development. Vol.50. P.31-46.

Manchenko G.P. 1994. Handbook of detection of enzymes on electrophoretic gels. Sydney: CRC Press, Inc. 341 p.

McLaughlin P.A., Asakura A. 2004. Reevaluation of the hermit crab genus Parapagurodes McLaughlin \& Haig, 1973 (Decapoda: Anomura: Paguroidea: Paguridae) and a new genus for Parapagurodes doederleini (Doflein, 1902) // Proceedings of the Biological Society of Washington. Vol.117. P.42-56.

McLaughlin P.A., Haig J. 1973. On the status of Pagurus mertensii Brandt, with descriptions of a new genus and two new species from California (Crustacea: Decapoda: Paguridae) // Bulletin of the Southern California Academy of Sciences. Vol.72. P.113-136.

McLaughlin P.A., Gore R.H. 1988. Studies on the provenzanoi and other pagurid groups: I. The larval stages of Pagurus maclaughlinae García-Gómez, 1982 (Decapoda: Anomura: Paguridae), reared under laboratory conditions // Journal of Crustacean Biology. Vol.8. P.262-282. 
McLaughlin P.A., Jensen G.C. 1996. A new species of hermit crab of the genus Parapagurodes (Decapoda: Anomura: Paguridae) from the Eastern Pacific, with a description of its first zoeal stage // Journal of Natural History. Vol.30. P.841-854.

Mia Md.Y., Fuseya R., Watanabe S. 1999. Genetic variations among the three species of grapsid crabs, Helice tridens latimera, $H$. tridens tridens and H. leachi // Crustacean Research. No.28. P.52-61.

Mulley J.C., Latter B.D.H. 1980. Genetic variation and evolutionary relationships within a group of thirteen species of penaeid prawns // Evolution. Vol.34. P.904-916.

Nei M. 1978. Estimation of average heterozygosity and genetic distance from a small number of individuals // Genetics. Vol.89. P.583-590.

Pudovkin A.I., Zaikin D.V., Tatarenkov A.N. 1996. DBOOT computer software for calculation of Neis genetic distance and genetic identity and their bootstrapped confidence intervals // Genetica. Vol.32. P.1017-1020.

Roberts M.H.J. 1970. Larval development of Pagurus longicarpus Say reared in the laboratory, I. Description of larval instars // Biological Bulletin. Vol.139. P.188-202.

Rohlf F.J. 1988. NTSYS-pc: Numerical taxonomy and multivariate analysis system. New York: Exter Publishing.
Stewart B.A., Cook P.A. 1998. Identification of a new species of river crab (Decapoda: Brachyura: Potamonautidae) from South Africa using morphological and genetic data // Journal of Crustacean Biology. Vol.18. P.556-571

Swofford D.L, Selander R.B. 1981. BIOSYS: A computer program for analysis of allelic variation in genetics. Urbana: Univ. of Illinois.

Thorpe J.P. 1982. The molecular clock hypothesis: biochemical evolution, genetic differentiation and systematics // Annual Review of Ecology and Systematics. Vol.13. P.139-168.

Thorpe J.P., Solé-Cava A.M. 1994. The use of allozyme electrophoresis in invertebrate systematics // Zoologica Scripta. Vol.23. P.3-18.

Zaslavskaya N.I. 1989. [Genetic variability in four Pacific species of periwinkles (Mollusca: Gastropoda)] // Genetika. Vol.25. P.1636-1644 [in Russian, with English summary].

Zaslavskaya N.I. Kornienko E.S., Korn O.M. 2007. Genetic differences between two spider crabs Pisoides bidentatus (A. Milne-Edwards, 1873) and Pugettia quadridens (de Haan, 1839) (Decapoda: Brachyura: Majoidea) from the Sea of Japan // Biochemical Systematics and Ecology. Vol.35. P.750-756. 